Sociologie et sociétés

SOCIOLOGIE

ET SOCIÉTÉS

\title{
La fin des corporations professionnelles au Québec
}

\section{Guy DEMERS}

Volume 2, numéro 2, novembre 1970

URI : https://id.erudit.org/iderudit/001176ar

DOI : https://doi.org/10.7202/001176ar

Aller au sommaire du numéro

Éditeur(s)

Les Presses de l'Université de Montréal

ISSN

0038-030X (imprimé)

1492-1375 (numérique)

Découvrir la revue

Citer cette note

DEMERS, G. (1970). La fin des corporations professionnelles au Québec.

Sociologie et sociétés, 2(2), 317-326. https://doi.org/10.7202/001176ar d'utilisation que vous pouvez consulter en ligne.

https://apropos.erudit.org/fr/usagers/politique-dutilisation/ 


\section{Note critique}

\section{La fin des corporations professionnelles au Québec}

Dans son rapport sur les Professions et la société, publié en juillet 1970 1, la Commission d'enquête sur la santé et le bien-être social (Commission Castonguay) propose une réforme en profondeur du droit qui régit les organisations professionnelles au Québec, leurs rapports réciproques et leurs rapports avec la société et avec l'État.

En raison des changements importants que cette réforme peut impliquer ou supposer pour l'ordre social, économique et politique québécois, le projet de réforme annoncé constitue pour le sociologue un objet d'analyse privilégié et en même temps une occasion unique de recherche et de réflexion.

Dans ce texte, nous préciserons tout d'abord la nature du projet de réforme dans le contexte actuel de l'organisation des services au Québec. Nous traiterons ensuite un peu plus longuement de la signification que la réforme pourrait avoir pour l'un des groupes professionnels les plus directement concernés - le groupe des médecins. Ceci nous permettra également de soulever la question d'une sociologie du changement organisationnel appliquée au monde des professions. Enfin, en guise de conclu-

1. Rapport de la Commission d'enquête sur la santé et le bien-être social, cinquième partie, vol. 7, t. I : les Professions et la société, Gouvernement du Québec, 1970. sion, nous soulèverons le problème de l'adaptation de l'appareil de l'État au rôle moteur nouveau qu'il est appelé à jouer dans le domaine de la planification et de l'organisation des services.

\section{LE PROJET DE RÉFORME}

Fondée sur une analyse critique extrêmement sévère du droit actuel des professions au Québec - \& il faut conclure, affirment notamment les auteurs du rapport (p. 27), que le droit des professions a été davantage l'expression de la force des groupes professionnels que la transcription des besoins sociaux et professionnels dans le droit $\gg$ - la réforme proposée consiste fondamentalement d'une part, au niveau de chaque profession, à reconnaître le principe du partage des fonctions de « représentation des intérêts» et d'《agent de la collectivité » traditionnellement exercées par les corporations professionnelles et, d'autre part, au niveau de l'ensemble des professions, à reconnaître le principe de l'égalité des professions devant la loi.

Au niveau de chaque profession, il s'agit en particulier d'une réforme radicale du droit des corporations professionnelles en vue de les transformer en de véritables «ordres professionnels » ou encore en de véritables organismes 
de service public décentralisés de l'État et chargés de la protection du public ${ }^{2}$.

Et il s'agit, parallèlement, à partir d'une reconnaissance juridique des associations ou syndicats professionnels et de leur rôle en tant que promoteurs et défenseurs des intérêts socio-économiques et professionnels de leurs membres, d'adopter un droit nouveau devant s'appliquer aux rapports économiques et professionnels de ces groupes les uns avec les autres et avec l'État en tant que représentant de la collectivité.

$\mathrm{Au}$ niveau de l'ensemble des professions, outre l'adaptation du droit aux besoins de la société actuelle et la recherche de cohérence dans l'ensemble des dispositions relatives aux professions, il s'agit d'adopter et de mettre en force le principe de la spécialisation fonctionnelle qui exclura l'immixtion d'un organisme professionnel dans le champ d'activité d'un autre et, par voie de conséquence, la hiérarchisation des professions.

Si l'on veut faire un peu d'histoire, cette réforme pourra apparaître, si elle est adoptée, comme la conséquence logique de toute autre série de décisions politiques qui ont marqué ces dernières années l'évolution des services au Québec et principalement l'organisation des services de santé. Nous nous référons ici à la prise en charge progressive par l'État non seulement de l'orientation et de la restructuration des services de santé et de leur organisation, mais en-

2. Comme service public [l'Ordre] assume un rôle politique dans le fonctionnement de l'Etát : 1) rôle gouvernemental, lorsqu'il est habilité à contrôler l'accès à l'exercice de la profession en lieu et place d'un diplôme conféré par le système d'enseignement public; 2) rôle législatif, lorsqu'il arrête certaines normes (règlements, codes d'éthique et de déontologie) relatives aux conditions d'exercice de la profession; 3) rôle administratif, lorsqu'il est chargé de l'inspection et de l'examen des actes professionnels; 4) rôle juridictionnel, enfin, lorsqu'il est chargé de juger et, au besoin, de sanctionner les actes ou les fautes contre les normes en usage. * (Op. cit., p. 18). core de la planification concertée et de la direction des services de santé.

Il conviendrait cependant d'analyser le phénomène d'une manière plus globale. Il serait extrêmement instructif de faire une analyse comparative de l'évolution de l'organisation des services de santé et de bien-être et de celle des autres services juridiques, techniques ou scientifiques et, surtout lorsqu'on observe les réactions des corps professionnels les plus directement visés, de noter les conséquences de la réforme pour les organisations professionnelles existantes dans chacun de ces domaines.

Ce n'est sans doute pas un hasard si l'auteur du projet de réforme est la Commission Castonguay sur la santé et le bien-être social. Mais il serait certainement intéressant à cet égard de comparer le rapport de la Commission Castonguay à celui d'une autre commission qui a soumis son rapport récemment, la Commission Prévost sur l'administration de la justice.

La réforme proposée pourrait également apparaître à l'analyse comme la conséquence logique d'un changement profond de la philosophie politique québécoise à l'égard du rôle de l'État dans l'organisation des services en général et notamment dans le domaine de la santé.

Car, en ce qui a trait aux services de santé, bien qu'aujourd'hui cela puisse paraître aller de soi, c'était déjà il y a dix ans et c'est encore aujourd'hui pour les principaux intéressés un changement majeur que celui de reconnaître à l'État le devoir et le droit de transformer l'ordre traditionnel libéral des rapports économiques dans le domaine de la santé - ordre fondé sur le mécanisme du marché libre des soins - au profit d'un ordre nouveau (cf. l'assurance-maladie) défini par le contrôle collectif du public consommateur, grâce notamment au mécanisme du tiers-payant ou du tiersresponsable, sur l'offre monétaire globale et sur la distribution des soins. 
Mais c'est maintenant un changement encore plus important de reconnaître que cette exigence sociale première doit engendrer un contrôle par l'État de la planification et de l'organisation des services de santé; d'affirmer que le contrôle collectif de la distribution des soins ne peut s'effectuer sans un contrôle collectif de la production ellemême; et de dire que la société doit choisir les secteurs d'action à développer en priorité et doit rationaliser et déterminer la contribution relative des divers agents institutionnels et professionnels de soins dans l'organisation des services de santé.

Ce changement (cf. la loi et les règlements des hôpitaux ainsi que le projet de réforme du droit des professions) ne peut manquer d'affecter non seulement les rapports entre les agents institutionnels et professionnels de soins et l'Etat, mais encore l'organisation elle-même des institutions et des professions.

\section{LA SIGNIFICATION DU PROIET DE RÉFORME POUR L'ORGANISATION PROFESSIONNELLE MÉDICALE}

La réforme annoncée du droit des professions pourrait apparaître également comme l'aboutissement logique de l'évolution récente de certaines organisations professionnelles et notamment de l'organisation professionnelle médicale. Car c'est un fait que celle-ci a subi de profondes transformations depuis la fin des années 50 , soit depuis que le syndicalisme a fait son apparition au sein de la profession. Et, n'eût-ce été ces transformations, le projet de réforme ne serait probablement pas apparu au moment où il a été annoncé et peut-être pas sous sa forme actuelle.

Il convient donc, si l'on veut comprendre la signification du projet pour l'un des groupes les plus directement concernés et prévoir quelque peu sa réaction, d'analyser les transformations qu'a subies sa propre organisation professionnelle et de chercher à identifier les facteurs sociaux, économiques et politiques qui ont pu historiquement les rendre possibles sinon nécessaires.

a) Les transformations majeures récentes de l'organisation professionnelle médicale au Québec. - Jusqu'à très récemment l'organisation professionnelle médicale québécoise pouvait être désignée comme une organisation «corporative» multifonctionnelle. Sur le plan structurel le corps médical québécois était gouverné par un appareil unique, le Bureau des gouverneurs du Collège des médecins, qui monopolisait toutes les fonctions tant au niveau de la promotion et du développement d'une médecine de qualité en vue du bien public, qu'à celui de la défense des intérêts individuels et collectifs des médecins. Depuis les années 50 toutefois, une certaine décentralisation des pouvoirs s'était effectuée, faisant apparaître des sousappareils quasi autonomes, notamment les bureaux médicaux des hôpitaux, tandis qu'étaient fondées les premières associations médicales. Sur le plan des règles du jeu décisionnel, outre l'élection générale des gouverneurs suivant une représentation régionale, il n'était guère reconnu officiellement d'autre mode de représentation et de participation au gouvernement de la profession que le mode individuel.

Mais avec l'apparition du syndicalisme èt depuis que le syndicalisme a été reconnu officiellement comme forme d'organisation et d'action au sein de la profession au début des années 60 , des changements majeurs sont survenus au sein de l'organisation professionnelle : 1) L'institution d'une structure nouvelle syndicale de gouvernement face à l'ancienne structure corporative qui continue de persister et la dépossession de 
certaines fonctions corporatives dévolues à l'ancienne structure au profit de la structure nouvelle; 2) Une différenciation des modes de gouvernement et de représentation faisant apparaître, face à l'appareil central corporatif, plusieurs appareils-directeurs syndicaux et, face à la représentation individuelle obligatoire au sein de la corporation professionnelle, un mode de représentation par groupe et volontaire au sein de la structure syndicale; 3) L'institution de fait, tant au niveau de la corporation qu'au niveau de la structure syndicale, de la règle de la «parité » de représentation et de pouvoir des groupes d'intérêts médicaux.

Ces changements, le projet de réforme du droit des professions semble vouloir les accentuer : 1) Les anciennes corporations vont désormais perdre leur nom et seront désignées comme des «Ordres »;2) Elles ne vont conserver que leurs attributions «ordinales》 ou de protection du bien public et ne pourront plus fixer le tarif d'honoraires (même à titre indicatif) de leurs membres non conventionnés lorsque leurs membres rendront des services généralement défrayés par l'État au titre d'une convention; 3 ) Leurs liens de dépendance vis-à-vis de l'État seront accentués et clarifiés et certaines de leurs attributions, normalement du ressort de l'État, reviendront à l'État, comme l'admission à l'étude et les diplômes; 4) Des membres nommés par le lieutenantgouverneur en conseil sur la recommandation des milieux d'enseignement concernés, des associations concernées, du public consommateur et des fonctionnaires des ministères intéressés s'ajouteront aux membres élus de la profession aux conseils des Ordres; 5) Un Code des professions constituant le régime $\mathrm{du}$ droit des occupations remplacera la mosaïque des lois relatives aux professions. Il comprendra notamment des dispositions visant le monopole d'un titre professionnel ou technique auparavant insérées dans les lois particulières des professions.

b) Les arguments avancés au sein de la profession pour justifier les changements initiaux. - Pour justifier l'institution d'une structure syndicale parallèle à la structure corporative (ou encore la différenciation au niveau des structures des fonctions corporatives ordinales et syndicales) les acteurs concernés ont invoqué plusieurs arguments.

Tout d'abord il y aurait l'antinomie des fonctions ordinales et syndicales, pourtant associées depuis toujours. La fonction ordinale, impliquant la promotion et la défense d'une médecine orientée vers le bien public, ne pourrait s'exercer pleinement sans s'opposer, du moins parfois, à la promotion des intérêts professionnels. Et la fonction syndicale, impliquant la promotion et la défense des intérêts professionnels, bien qu'elle ne doive pas moralement faire fi du bien public, ne pourrait, elle aussi, s'exercer pleinement sans mettre en cause, du moins parfois, le bien public. Les hommes étant ce qu'ils sont, on ne peut logiquement vouloir réaliser l'une et l'autre fonction conjointement sans favoriser l'une ou l'autre.

Ensuite il y aurait, sur le plan stratégique, une « déséconomie » à maintenir une structure corporative multifonctionnelle. En effet les médecins, qui tiennent à une médecine de qualité et qui ont la responsabilité collective de la défendre et de la développer, ne pourraient de fait que discréditer l'autorité de l'appareil unique corporatif si cet appareil, en voulant négocier leurs intérêts, ne pouvait que donner l'impression de faire passer les intérêts professionnels en premier. En outre l'appareil corporatif étant une créature de jure de l'État, son pouvoir étant un pouvoir décentralisé de l'État, il ne pourrait pas employer certains moyens de pression 
(en particulier la grève ou la menace de grève), et donc défendre avec efficacité en toute situation les intérêts de la profession, sans risquer de perdre ce pouvoir délégué jugé nécessaire à une bonne organisation de la médecine.

D'un autre côté, pour justifier l'institution d'une structure syndicale «multipolaire (les fédérations syndicales), et pour justifier la règle de la parité de représentation des groupements d'intérêtsi, tant au niveau de la structure corporative qu'à celui de la structure syndicale, les acteurs concernés apportent de nouveaux arguments : en premier lieu ils invoquent les différences d'intérêts de leurs membres, le fait que ces intérêts ont le droit de s'associer et le fait qu'étant liés à des situations différentes face à l'environnement il est nécessaire que leurs membres appartiennent à des organisations d'action spécifiques; en second lieu ils invoquent les oppositions entre les groupements d'intérêts qui engendrent nécessairement des difficultés d'ajustement ou de conciliation, et ils invoquent le fait que, dans une structure de représentation suivant la règle de la majorité, ces intérêts rivaux ou opposés ne pourraient être adéquatement représentés. La majorité ne peut pas toujours respecter la minorité, surtout et encore moins se déposséder à son profit.

c) Des arguments aux hypothèses d'analyse. - Les explications fournies par les acteurs qui ont vécu ou qui ont fabriqué les changements peuvent et doivent constituer le point de départ d'une analyse des facteurs qui ont pu faire apparaître ces changements. Mais c'est en tant qu'hypothèses à vérifier que ces explications doivent être considérées par le chercheur. De plus, les arguments justificateurs n'expliquent pas tout. Certaines questions restent en suspens.

Comment par exemple ne s'est-on pas rendu compte plus tôt de l'antinomie des fonctions et des déséconomies qu'il y a à les réunir au sein d'une même structure? De quelle façon, en outre, cette prise de conscience a-t-elle pu effectivement engendrer les changements observés ? Comment se fait-il aussi que des intérêts opposés aient pu émerger au sein de la profession? Pourquoi ont-ils pu être obligés de rechercher une autre forme de gouvernement que celui de la majorité ? Et comment a-t-il été possible enfin aux divers intérêts de se grouper (ou de se mobiliser) d'une manière autonome et d'exiger la parité des voix aussi bien au sein de la structure corporative qu'au sein de l'intersyndicale?

1. Les facteurs internes. - On a dit que les changements observés pouvaient être liés au bon fonctionnement de l'organisation professionnelle, d'une part à sa capacité de résoudre effectivement les contradictions entre les objectifs ordinaux et syndicaux, et d'autre part à sa capacité de représenter des intérêts différents et de concilier et d'arbitrer les rivalités.

C'est un fait que la médecine a beaucoup évolué au Québec durant ces vingt dernières années. Au niveau de son contenu, on a observé une différenciation progressive des tâches, tout d'abord entre une pratique générale et une pratique spécialisée, ensuite entre des pratiques spécialisées du diagnostic et du traitement, enfin entre la pratique et l'exercice de l'enseignement et de la recherche.

Au niveau des rapports de travail on a observé une concentration des moyens et des technologies (en raison de leur spécialisation, mais aussi de leur coût) et une certaine coordination du travail (en raison de l'émergence d'une exigence accrue d'efficacité dans la communication des informations et des innovations et dans la consultation) : d'où l'apparition d'équipes médicales et 
d'une certaine hiérarchisation des fonctions et responsabilités.

Enfin, au niveau de l'organisation des services médicaux, l'hôpital d'une part, l'université d'autre part, sont devenus, à côté des bureaux privés ou groupés, des centres indispensables pour l'exercice de la médecine, faisant apparaître un type nouveau de médecin : le fonctionnaire ou le plein-temps hospitalier ou universitaire.

Si l'on veut comprendre les forces internes qui ont pu faire apparaître le syndicalisme au sein de la profession, il faut donc se demander d'abord dans quelle mesure cette évolution de la médecine a pu engendrer des exigences nouvelles, aussi bien en ce qui a trait à son orientation au bien public qu'en ce qui concerne les intérêts de la profession, et dans quelle mesure ces exigences ont pu effectivement être contradictoires ou encore être telles qu'il était impossible à l'organisation corporative traditionnelle de les rencontrer sans favoriser les unes au détriment des autres.

On peut prendre pour exemple l'exigence d'une distribution géographique équilibrée des travailleurs médicaux entre les grandes villes et les régions périphériques et se demander dans quelle mesure la promotion de l'intérêt d'une médecine hospitalière et spécialisée concentrée dans les grands centres pouvait s'allier à une exigence de revalorisation d'une médecine générale déconcentrée.

La prise de conscience de l'antinomie des fonctions corporatives syndicales et ordinales et la prise de conscience des déséconomies du maintien d'une structure corporative multifonctionnelle n'ont pas été non plus un phénomène subit et général. Il faut donc chercher à voir qui en a pris conscience, à quel moment, en raison de quelle expérience et pourquoi ceux-là en ont pris cons- cience et non pas ceux-ci. Certains médecins, ou certains groupes de médecins, ont pu être dans des situations telles qu'ils ont pu faire l'expérience à leurs dépens des contradictions entre les objectifs ordinaux et syndicaux et des déséconomies de vouloir promouvoir les uns et les autres au sein d'une même structure.

Mais cela ne suffit pas encore à expliquer l'émergence du syndicalisme chez les médecins québécois. Le syndicalisme a d'abord été un regroupement et une mobilisation de fait de certains éléments ou de certains intérêts : d'abord le fait d'un regroupement chez certains médecins spécialistes, puis le fait d'un regroupement de masse chez les omnipraticiens. Il est donc permis de se demander pourquoi cela s'est produit ainsi et de chercher si la raison principale de ce syndicalisme de fait était l'expérience de l'antinomie et des déséconomies dont on a parlé ou bien si elle était l'expérience de quelque chose d'autre, comme celle d'intérêts opposés ne pouvant se réaliser pleinement au sein de la structure corporative traditionnelle.

Il faut alors revenir à l'évolution de la médecine et se demander en quoi et comment cette évolution a pu directement ou indirectement faire apparaître des groupes d'intérêts spécifiques et même opposés. On affirme qu'il s'est constitué, au sein de la profession, un phénomène de classe. Dans quelle mesure une telle affirmation peut-elle être vérifiée ? Et même si cela était vrai, cela n'expliquerait pas nécessairement l'émergence. d'une structure syndicale multipolaire et l'institution de facto de la parité de représentation des groupes d'intérêts.

On peut s'interroger sur la représentativité de l'organisation corporative traditionnelle et sur sa capacité de résoudre les conflits d'intérêts au sein de la profession et même les conflits 
dans les définitions de l'orientation de la médecine moderne en fonction du bien public.

Les faits démontrent enfin que les premières associations furent des associations de médecins spécialistes. Comment pouvons-nous expliquer l'émergence de ces associations au cours des années 50 ? Comment se sont-elles définies les unes par rapport aux autres? Quels ont été leurs rapports avec les autres médecins non associés à ce moment-là, notamment les omnipraticiens? Et quel a été leur rôle dans le changement? Les faits prouvent que ce sont les omnipraticiens qui ont mené la bataille pour la reconnaissance du syndicalisme au sein de la profession. Pourquoil eux et non les autres ?

Finalement il faudrait connaître de quelle façon le syndicalisme de fait a pu réussir à se constituer, malgré plusieurs contraintes, dont celle de l'unité professionnelle; ensuite établir comment, peu après, a pu être admise officiellement la formule syndicale au sein de l'ensemble du corps médical et avec quelles nuances dans sa définition. Il a bien fallu convaincre et influencer.

2. Les facteurs externes. - L'organisation professionnelle des médecins québécois se serait-elle transformée de la façon que nous savons s'il n'y avait eu que ce jeu des forces internes? Sans doute ne pourra-t-on répondre entièrement à une telle question. Mais on a pu constater que l'émergence du syndicalisme a coïncidé dans le temps avec un changement majeur dans les rapports $\mathrm{du}$ corps médical avec son environnement, et notamment avec l'État.

Depuis la fin des années 50 , un changement majeur s'est produit au Québec dans les rapports du corps médical et de l'État. Ce changement, on l'a défini par la prise en charge progressive par l'État de la planification et de la direction de l'organisation des services de santé. On peut penser que l'apparition de cette volonté de réforme dans le domaine de la santé, qui s'est manifestée dès 1959 , sous le gouvernement Sauvé, et qui n'a fait que se préciser par la suite jusqu'à aujourd'hui, a pu changer profondément la situation traditionnelle du corps médical.

En tant que producteur de soins et en tant que demandeur d'une certaine rémunération, la situation relative du corps médical québécois comparée à celle des autres agents institutionnels et professionnels de soins a été réévaluée. La qualité de soins à produire, leur distribution, ont été mises en question. Et que dire du niveau de rémunération des médecins par rapport à celui des autres agents professionnels ?

Mais il n'y a pas que la situation de producteur et de demandeur qui a changé; il y a aussi celle du corps médical face aux autres agents institutionnels et professionnels, au sein du système de prise de décision global dans le secteur de la santé. Tout d'abord il y a eu l'émergence de ce nouveau partenaire, l'État, qui n'est plus seulement « arbitre », mais qui décide. Ensuite il y a eu une certaine formalisation de la consultation et de la discussion où le corps médical n'était plus assuré autant qu'avant d'une position dominante.

Plus encore, dans la mesure où il $a$ été et où il est encore question d'une réorganisation globale du système de production interprofessionnel, de l'élimination des hiérarchies professionnelles et de l'institution d'appareils-directeurs interprofessionnels ayant autorité sur l'ensemble des services de santé, on a pu se demander à juste titre si la place du corps médical comme entité autonome fonctionnelle ne serait pas ellemême remise en question.

En quoi ces changements, tels qu'ils se sont présentés concrètement et tels 
qu'ils se sont manifestés dans les intentions avouées des acteurs concernés, notamment des gouvernants et de leurs conseillers, ont-ils pu faire apparaitre l'antinomie des fonctions ordinales et syndicales et les déséconomies de maintenir une organisation professionnelle multifonctionnelle?

Était-il nécessaire que la Commission Castonguay formule justement son projet de réforme du droit des professions au moment où l'Assemblée nationale s'apprêtait à adopter la Loi de l'assurance-maladie ? Était-ce là pure coïncidence? De toute façon, il faudrait savoir si le projet de réforme vient classer une affaire ou relancer le processus du changement de l'organisation professionnelle médicale.

Enfin, il est permis de se demander aujourd'hui si la série des décisions politiques des dix dernières années touchant le domaine des services de santé a pu modifier la situation relative des groupes d'intérêts au sein de la profession et rendre peut-être plus difficile la conciliation des intérêts, en accordant par exemple un support direct ou indirect à l'action autonome de certains groupes. Ces décisions ont-elles eu pour conséquence un affaiblissement de l'unité professionnelle chez les médecins? Dans cette perspective, devra-t-on conclure que la décision de réforme du droit des professions a été, dans la mesure où l'on considère la profession médicale, volontairement ou involontairement préparée de longue main par une série d'actions ou de décisions politiques qui ont eu pour effet de diviser le corps médical et de rendre l'organisation professionnelle médicale plus facilement maniable?

d) La réaction probable du corps médical au projet de réforme. - L'histoire à faire nous permettra de mieux évaluer l'impact probable réel de la réforme. Car, est-il besoin de le souli- gner, sans nier son importance, il ne s'agit ici que d'une réforme du «droit». Une telle réforme ne signifie pas nécessairement ou immédiatement la transformation radicale des organisations et des rapports que le droit doit régir. A ce sujet on peut encore citer les auteurs du rapport : « Le grand problème actuel $\mathrm{du}$ droit et de l'organisation professionnels, c'est son inadaptation. Non seulement le droit professionnel n'exprimet-il plus les besoins de la société moderne, mais encore il va souvent à l'encontre même des conceptions idéologiques et techniques qui y prévalent et qui président à son évolution » (p. 23).

Il n'est pas du tout certain que les changements de mentalité des groupes impliqués et que les transformations de leurs rapports de pouvoir aient été suffisants et assez profonds, compte tenu des changements observés, pour qu'il soit plausible d'attendre d'eux une acceptation réelle, dans leurs actions concrètes, de la philosophie sous-jacente à la réforme proposée. Pour se convaincre des difficultés à surmonter, il suffit de réfléchir sur la réaction persistante d'une partie importante du corps médical devant la Loi de l'assurance-maladie adoptée par l'Assemblée nationale en juillet 1970. Et il serait tout aussi instructif d'analyser les réactions des autres corps professionnels face à la réforme, celles du Barreau par exemple.

La transformation des anciennes corporations n'interdit pas aux associations professionnelles d'adopter une attitude "corporative» et pour les groupes qui sont gratifiés du statut et des privilèges d'une profession libérale, de vouloir les maintenir et, l'histoire se répétant en reprenant les termes mêmes de l'analyse critique présentée dans le rapport (p. 28) - de commencer «par requérir l'incorporation en association volontaire », puis de demander «l'adhésion 
obligatoire avec contrôle des conditions d'admission» pour finir «par arracher la réglementation des conditions d'exercice et le contrôle de l'activité qui en découle ».

e) Pour une sociologie de l'organisation professionnelle face au changement. - Il est plus facile d'énumérer une série d'hypothèses pour expliquer un changement comme celui qui touche l'organisation professionnelle médicale que d'élaborer un modèle d'analyse sociologique pertinent pour l'étude des processus du changement, de l'appliquer et d'en démontrer l'utilité théorique et pratique. La description sommaire que nousi avons faite des transformations de l'organisation professionnelle médicale au Québec et celle d'un certain nombre de questions qui demeurent inexplorées touchant ces transformations, justifient suffisamment la recherche à faire dans ce sens. Nous pensons en particulier à l'intérêt non équivoque qu'il y aurait à poursuivre, dans le contexte des organisations professionnelles, l'élaboration et l'application du modèle d'analyse du changement organisationnel tel que conçu par le sociologue Bernard Poisson ${ }^{3}$. Étant donné les implications très vastes de la réforme du droit qui régit les organisations professionnelles au Québec - bien qu'il s'agisse en réalité d'une réforme des composantes «juridiques » des appareils organisationnels - un tel travail devrait déboucher rapidement sur une analyse comparative des processus de changement dans un ensemble complexe d'organisations professionnelles. Ce pourrait être le point de départ véritable d'une sociologie des organisations professionnelles au Québec. Une occasion à ne pas manquer.

3. Bernard Poisson, Schéma d'analyse de ll'organisation religieuse $*$, Sociologie et Sociétés, vol. $1, \mathrm{n}^{\circ} 2$, novembre 1969 , p. 147 170.

\section{L'ADAPTATION NÉCESSAIRE \\ DE L'APPAREIL DE L'ÉTAT}

Traditionnellement, le rôle de l'État, au Québec, se limitait à garantir les libertés individuelles et l'ordre juridique. Amené à intervenir dans le domaine économique et social à titre d'agent planificateur et «organisateur», il lui faut désormais effectuer des choix qui vont influencer la vie de l'ensemble de la société et les imposer avec autorité.

Mais l'Etat ne peut avoir le monopole de la rationalité et de l'information pertinente. S'il est important qu'il obtienne un pouvoir de décision et d'arbitrage, ce pouvoir n'aura sa pleine efficacité que dans la mesure où les acteurs concernés pourront d'une part être associés ou consultés lors de l'élaboration des projets collectifs et, d'autre part, contester les activités de l'État en ces mêmes matières. 11 est extrêmement important de tenir compte de ces données lorsqu'on veut analyser l'impact probable d'un projet de réforme comme celui du droit des organisations professionnelles. En prenant en charge l'orientation, la planification, l'organisation, la direction des services, comme c'est le cas dans le domaine de la santé et du bien-être, comme ce peut l'être dans d'autres domaines, l'État est lui aussi appelé à se transformer.

Les rapports politiques traditionnels doivent être transformés non seulement quant à l'objet du pouvoir de l'État, mais encore quant aux formes mêmes et aux processus d'élaboration, de décision, de mise en ceuvre et de contrôle des politiques de l'État. Le jeu démocratique traditionnel doit changer. Il ne peut plus être limité à l'intérieur d'un contexte social et d'un ordre légal donné - d'ailleurs en changement - à la lutte pour l'accession au pouvoir de gouverner. Il devient nécessaire que le contenu même des décisions politi- 
ques soit objet de discussion et de sanction et cela d'une manière permanente.

La concertation des décisions de l'État ne signifie pas une transformation de celui-ci en un vaste système de cogouvernement, ni le remplacement du système traditionnel de désignation des gouvernants par un autre système fondé sur la participation exclusive des corps intermédiaires. La concertation ne garde son sens que si les partenaires demeurent identifiables et identifiés. Elle s'oppose autant à l'intégration des partenaires économiques et sociaux de l'État - les professionnels par exemple - à l'appareil de l'État qu'à la disparition du politique dans le social et l'économique.

Si l'on doit dire que c'est dans le dynamisme des relations de l'appareil de l'État (et de ses composantes) avec ses environnements qu'il faut chercher les sanctions positives et négatives nécessaires à la rationalité même de son action responsable, il faudrait pouvoir vérifier si ces sanctions existent vraiment. Dans la mesure où la concertation doit être autre chose qu'un jeu de lobbying - on sait que le lobbying porte généralement sur les points faibles de l'appareil de l'État et qu'il cherche à élaborer une stratégie en bonne partie secrète - elle exige la création de canaux institutionnels et obligatoires de communication entre l'appareil de l'État à tous ses niveaux et ses partenaires économiques et sociaux.

Compte tenu des changements auxquels la réforme du droit des professions est liée, une question fondamentale doit en définitive être également posée : l'État est-il apte à relever le défi de cette société de concertation que, pourtant, il contribue à créer ?

GUY DEMERS 